Geometry 85 Topology

Volume 5 (2001) 335-345

Published: 2 April 2001

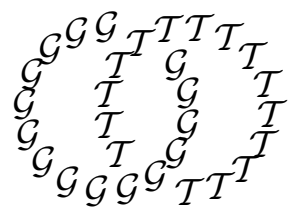

\title{
Complex cobordism of involutions
}

\author{
N P STRICKLAND \\ Department of Mathematics, University of Sheffield \\ Western Bank, Sheffield, S10 2TN,UK \\ Email: N.P.Strickland@sheffield.ac.uk
}

\begin{abstract}
We give a simple and explicit presentation of the $\mathbb{Z} / 2$-equivariant complex cobordism ring, as an algebra over the nonequivariant complex cobordism ring.
\end{abstract}

AMS Classification numbers Primary: 55N22

Secondary: $55 \mathrm{~N} 91$

Keywords: Equivariant, complex cobordism, involution

Proposed: Haynes Miller

Seconded: Ralph Cohen, Gunnar Carlsson

(c) Geometry $\&$ Topology Publications
Received: 20 February 2001

Revised: 4 March 2001 


\section{Introduction}

Let $A$ be the group of order two, and let $M U_{A}^{*}$ be the homotopical $A$-equivariant complex cobordism ring, as defined in [3] (for example). In this note we give a simple and explicit presentation of $M U_{A}^{*}$ as an algebra over the nonequivariant complex cobordism ring $M U^{*}$ (which is of course well understood).

Our construction and proofs are short and elementary so we have decided to publish them as they stand. Elsewhere, we will describe the conceptual background (formal multicurves and equivariant formal groups) and generalise our results to other compact Lie groups. In the general abelian case, we hope to be almost as explicit as in the present work. We will also describe $M U_{A}^{*} X$ for many naturally-occurring $A$-spaces $X$, using the algebraic geometry of formal schemes; from the right viewpoint, the results are closely parallel to their nonequivariant analogs. A large part of the theory depends only on the fact that $M U_{G}^{*} P V$ is a finitely generated projective module over $M U_{G}^{*}$ for all representations $V$, which holds when $G$ is abelian; we do not know whether there are any nonabelian examples.

Our approach is based on a pullback square exhibited by Kriz [2]. We note that Sinha [4] has also calculated $M U_{A}^{*}$, but his presentation is less explicit and depends on some choices.

\section{The model}

Let $L=M U^{*}$ be the Lazard ring, with universal formal group law $x+{ }_{F} y=$ $\sum_{i j} a_{i j} x^{i} y^{j}$. Recall that

$$
\begin{aligned}
& a_{i j}=a_{j i} \\
& a_{0 i}=\delta_{1 i}= \begin{cases}1 & \text { if } i=1 \\
0 & \text { otherwise }\end{cases}
\end{aligned}
$$

There are of course other relations, expressing the associativity of $F$. It is wellknown that $L$ can be expressed as a polynomial algebra $\mathbb{Z}\left[x_{1}, x_{2}, \ldots\right]$, although there is no system of generators that is both explicit and convenient. Here we take the structure of $L$ as given and concentrate on describing $M U_{A}^{*}$ as an algebra over it.

Let $R$ be generated over $L$ by elements $s_{i j}$ (for $i, j \geq 0$ ) and $t_{i}$ (for $i \geq 0$ ) subject to relations given below. We use $b_{k}$ as a synonym for $s_{0 k}$, and $e$ as a 
synonym for $b_{0}=s_{00}$. The relations are:

$$
\begin{aligned}
t_{0} & =0 \\
s_{10} & =1 \\
s_{i 0} & =0 \quad \text { for } i>1 \\
t_{k}-b_{k} & =e t_{k+1} \\
s_{j k}-a_{j k} & =e s_{j+1, k}
\end{aligned}
$$

We can give $R$ a grading with $\left|s_{i j}\right|=\left|a_{i j}\right|=2(1-i-j)$ and $\left|t_{k}\right|=2(1-k)$ so $\left|b_{k}\right|=2(1-k)$ and $|e|=2$.

Note that the equation $t_{0}-b_{0}=e t_{1}$ gives $\left(1+t_{1}\right) e=0$. Moreover, we have $t_{1}=b_{1}+e t_{2}=a_{01}+e s_{11}+e t_{2}=1+e\left(s_{11}+t_{2}\right)$, so $1-t_{1}=0(\bmod e)$. It follows that $1-t_{1}^{2}=\left(1-t_{1}\right)\left(1+t_{1}\right)=0$, so $t_{1}^{2}=1$.

Our main result is as follows:

Theorem 1 There is an isomorphism $R \simeq M U_{A}^{*}$ of graded $M U^{*}$-algebras.

The rest of this paper constitutes the proof.

Remark 2 Greenlees has studied the ring $L_{A}$ that classifies $A$-equivariant formal group laws; he shows that there is a surjective map $\alpha: L_{A} \rightarrow M U_{A}^{*}$ whose kernel is $e$-divisible and $e$-torsion (and is conjectured to be zero). One can deduce from the above theorem that there is a ring map $\beta: R=M U_{A}^{*} \rightarrow$ $L_{A}$ with $\alpha \beta=1$. It seems likely that our methods can be used to prove that $\alpha$ is an isomorphism, but we have not succeeded as yet.

\section{The pullback square}

Put $R^{\prime}=L\left[b_{0}, b_{1}, b_{2}, \ldots\right]\left[b_{0}^{-1}\right]$. Next, we define as usual $[2](x)=x+{ }_{F} x=$ $\sum_{i, j} a_{i j} x^{i+j} \in L \llbracket x \rrbracket$, and we put $\widehat{R}=L \llbracket e \rrbracket /[2](e)$, and $(\widehat{R})^{\prime}=\widehat{R}[1 / e]$. There is an evident map $\widehat{R} \rightarrow(\widehat{R})^{\prime}$, which we call $\zeta$. Next, we define a map $\xi: R^{\prime} \rightarrow(\widehat{R})^{\prime}$ by letting $\xi\left(b_{i}\right)$ be the coefficient of $x^{i}$ in the power series $x+{ }_{F} e$. (In particular, $\xi\left(b_{0}\right)=e$, which is invertible in $(\widehat{R})^{\prime}$, as required.)

The following result summarises much of what was previously known about $M U_{A}^{*}$ :

Geometry 83 Topology, Volume 5 (2001) 
Theorem 3 There is a pullback square as follows:

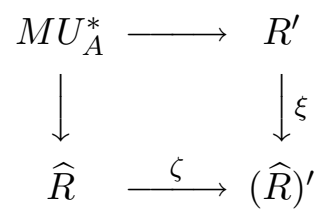

Proof This is Theorem 1.1 of [2], in the case $p=2$. Our element $b_{i}$ is Kriz's $b_{1}^{(i)} u_{1}$, and his $u_{1}$ is our $e$. There is a slight misprint in the statement: the element $b_{k}^{(0)}$ is 1 , not 0 . Geometrically, the square comes from the Tate diagram of cofibrations, as studied in [1]. In this context, $\widehat{R}$ is identified with $M U^{*} B A$, and $R^{\prime}$ with $\pi_{-*} \Phi^{A} M U_{A}$.

Below we shall exhibit a similar pullback square with $M U_{A}^{*}$ replaced by $R$; Theorem 1 follows by uniqueness of pullbacks. To show that our square is a pullback, we use the following result.

Theorem 4 Let $S$ be a ring, and $d$ an element of $S$. Suppose that $S$ has bounded $d$-torsion, or in other words that $\bigcup_{k} \operatorname{ann}_{S}\left(d^{k}\right)=\operatorname{ann}_{S}\left(d^{N}\right)$ for some $N$. Then there is a pullback square as follows:

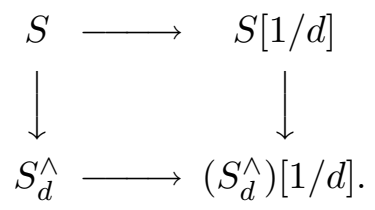

Proof This is standard, but we give a proof for completeness. First, it is clear that there is a commutative square as shown. Thus, if we let $T$ denote the pullback of $S[1 / d]$ and $S_{d}^{\wedge}$, we get a map $S \rightarrow T$, and we must show that it is an isomorphism.

Suppose that $u \in S$ has image zero in both $S[1 / d]$ and in $S_{d}^{\wedge}$. As $u \mapsto 0$ in $S[1 / d]$ we have $d^{k} u=0$ for some $k \geq 0$. As $u \mapsto 0$ in $S_{d}^{\wedge}=\lim _{\leftarrow} S / d^{j}$ we see that $u \mapsto 0$ in $S / d^{N}$, so $u=d^{N} v$ say. It follows that $d^{N+k} v=0$ but $\operatorname{ann}_{S}\left(d^{N+k}\right)=\operatorname{ann}_{S}\left(d^{N}\right)$ so $d^{N} v=0$ or in other words $u=0$. This means that the map $S \rightarrow T$ is injective.

Now suppose we have an element $(u, v) \in T$, so $u \in S[1 / d]$ and $v \in S_{d}^{\wedge}$, and $u$ and $v$ have the same image in $\left(S_{d}^{\wedge}\right)[1 / d]$. We then have $u=u^{\prime} / d^{i}$ for some $u^{\prime} \in S$ and $i \geq 0$, and $d^{j} u^{\prime}=d^{i+j} v$ in $S_{d}^{\wedge}$ for some $j \geq 0$. Next, we can choose a sequence $\left(v_{m}\right)$ in $S$ such that $v_{m}=v_{m+1}\left(\bmod d^{m}\right)$ and $v_{m} \rightarrow v$ in 
$S_{d}^{\wedge}$. It follows that the element $d^{j}\left(u^{\prime}-d^{i} v_{m}\right)=d^{i+j}\left(v-v_{m}\right)$ maps to zero in $S / d^{i+j+m}$, say $d^{j}\left(u^{\prime}-d^{i} v_{m}\right)=d^{i+j+m} w_{m}$. We may replace $v_{m}$ by $v_{m}+d^{m} w_{m}$ and thus assume that $d^{j} u^{\prime}=d^{i+j} v_{m}$ for all $m$. Now let $x_{m}$ be such that $v_{m+1}-v_{m}=d^{m} x_{m}$. As $d^{i+j} v_{m}=d^{j} u^{\prime}=d^{i+j} v_{m+1}$, we see that $d^{i+j+m} x_{m}=0$. As $\operatorname{ann}_{S}\left(d^{k}\right) \subseteq \operatorname{ann}_{S}\left(d^{N}\right)$ for all $k$, we have $d^{N} x_{m}=0$. When $m \geq N$ this gives $d^{m} x_{m}=0$ and thus $v_{m}=v_{m+1}$. We put $v^{\prime}=v_{N}=v_{N+1}=\ldots \in S$. It is clear that $v^{\prime} \mapsto v$ in $S_{d}^{\wedge}$. Moreover, the equation $d^{j} u^{\prime}=d^{i+j} v_{m}$ shows that $v^{\prime} \mapsto u^{\prime} / d^{i}=u$ in $S[1 / d]$, so $v^{\prime} \mapsto(u, v) \in T$. Thus, the map $S \rightarrow T$ is also surjective, as required.

It will be a significant part of our task to check that $R$ has bounded $e$-torsion.

\section{Completion at $e$}

The following proposition can be proved easily by reading the relations modulo $e$. Here $\delta_{1 k}$ is Kronecker's delta.

Proposition 5 There is a map $\epsilon: R \rightarrow L$ with

$$
\begin{aligned}
\epsilon\left(s_{i j}\right) & =a_{i j} \\
\epsilon\left(t_{k}\right) & =\epsilon\left(b_{k}\right)=\delta_{1 k} \\
\epsilon(e) & =0,
\end{aligned}
$$

and this induces an isomorphism $R / e \rightarrow L$.

This can be improved as follows:

Proposition 6 There is a map $\phi: R \rightarrow \widehat{R}$ given by

$$
\begin{aligned}
\phi\left(s_{i j}\right) & =\sum_{k \geq 0} a_{i+k, j} e^{k} \\
\phi\left(t_{k}\right) & =\sum_{i, j \geq 0} a_{i+k, j} e^{i+j} \\
\phi\left(b_{l}\right) & =\sum_{m \geq 0} a_{l m} e^{m} \\
\phi(e) & =e .
\end{aligned}
$$

If $\epsilon^{\prime}: \widehat{R} \rightarrow L$ is given by $\epsilon^{\prime}(e)=0$, then $\epsilon^{\prime} \phi=\epsilon$. Moreover, $\phi$ induces an isomorphism $\hat{\phi}: R_{e}^{\wedge} \rightarrow \widehat{R}$. 
Proof First, note that the definitions of $\phi\left(b_{l}\right)$ and $\phi(e)$ are simply the appropriate specialisations of the definition of $\phi\left(s_{i j}\right)$ so they can essentially be ignored.

To see that $\phi$ respects the relations, note that

$$
\begin{aligned}
\phi\left(t_{0}\right) & =\sum_{i j} a_{i j} e^{i+j}=[2](e)=0 \\
\phi\left(s_{10}\right) & =\sum_{k \geq 0} a_{1+k, 0} e^{k}=\sum_{k \geq 0} \delta_{k 0} e^{k}=1 \\
\phi\left(t_{k}\right)-\phi\left(b_{k}\right) & =\sum_{i>0} \sum_{j \geq 0} a_{i+k, j} e^{i+j} \\
& =e \sum_{i, j \geq 0} a_{i+k+1, j} e^{i+j}=e \phi\left(t_{k+1}\right) \\
\phi\left(s_{i j}\right)-a_{i j} & =\sum_{k>0} a_{i+k, j} e^{k} \\
& =e \sum_{k \geq 0} a_{i+k+1, j} e^{k}=e \phi\left(s_{i+1, j}\right) .
\end{aligned}
$$

It is trivial to check that $\epsilon^{\prime} \phi=\epsilon$.

There is an evident map $\psi: L[e] \rightarrow R$, which induces a map $\hat{\psi}: L \llbracket e \rrbracket \rightarrow R_{e}^{\wedge}$. As $\widehat{R}$ is already complete at $e$, we also get a map $\hat{\phi}: R_{e}^{\wedge} \rightarrow \widehat{R}$. It is easy to see that the composite $\hat{\phi} \hat{\psi}: L \llbracket e \rrbracket \rightarrow \widehat{R}=L \llbracket e \rrbracket /[2](e)$ is just the usual quotient map.

Next, one checks by induction on $m$ that in $R$ we have

$$
\begin{aligned}
s_{i j}-\sum_{l<m} a_{i+l, j} e^{l} & =s_{i+m, j} e^{m} \\
t_{k}-\sum_{l<m} b_{k+l} e^{l} & =t_{k+m} e^{m} .
\end{aligned}
$$

In each case the right hand side converges $e$-adically to 0 , so in $R_{e}^{\wedge}$ we have

$$
\begin{aligned}
s_{i j} & =\sum_{l} a_{i+l, j} e^{l} \\
t_{k} & =\sum_{l} b_{k+l} e^{l} .
\end{aligned}
$$

Using this, we see that $\hat{\psi}$ is surjective. Moreover, as a special case of the first equation, we have $b_{j}=\sum_{l} a_{l j} e^{l}$. Putting this into the second equation gives $t_{k}=\sum_{l, m} a_{k+l, m} e^{l+m}$. In particular, we have $[2](e)=\sum_{l, m} a_{l m} e^{l+m}=t_{0}=0$, 
so $\hat{\psi}$ factors through a map $\bar{\psi}: \widehat{R}=L \llbracket e \rrbracket /[2](e) \rightarrow R_{e}^{\wedge}$. As $\hat{\psi}$ is surjective and $\hat{\phi} \bar{\psi}=1$ we see that $\hat{\phi}$ is an isomorphism.

\section{Inverting $e$}

Proposition 7 There is a map $\theta: R \rightarrow R^{\prime}$ given by

$$
\begin{aligned}
& \theta\left(s_{i j}\right)=b_{j} b_{0}^{-i}-\sum_{l=1}^{i} a_{i-l, j} b_{0}^{-l} \\
& \theta\left(t_{k}\right)=-\sum_{l=1}^{k} b_{k-l} b_{0}^{-l}
\end{aligned}
$$

Moreover, this induces an isomorphism $R[1 / e] \rightarrow R^{\prime}$.

Proof First observe that $\theta\left(s_{0 j}\right)=b_{j}$ so the notation is self-consistent.

To see that $\theta$ respects the relations, note that

$$
\begin{aligned}
\theta\left(t_{0}\right) & =0 \\
\theta\left(s_{10}\right) & =b_{0} b_{0}^{-1}-a_{00} b_{0}^{-1}=1 \\
\theta\left(s_{i 0}\right) & =b_{0}^{1-i}-\sum_{l=1}^{i} a_{i-l, 0} b_{0}^{-l} \\
& =b_{0}^{i-1}-\sum_{l=1}^{i} \delta_{i-l, 1} b_{0}^{-l} \\
& =0 \quad \text { for } i>1 \\
\theta\left(t_{k}\right)-\theta\left(b_{k}\right) & =-b_{k}-\sum_{l=1}^{k} b_{k-l} b_{0}^{-l} \\
& =-\sum_{l=0}^{k} b_{k-l} b_{0}^{-l} \\
& =b_{0}\left(-\sum_{m=1}^{k+1} b_{k+1-m} b_{0}^{-m}\right) \\
& =b_{0} \theta\left(t_{k+1}\right)
\end{aligned}
$$

Geometry \& Topology, Volume 5 (2001) 


$$
\begin{aligned}
\theta\left(s_{i j}\right)-a_{i j} & =b_{j} b_{0}^{-i}-\sum_{l=0}^{i} a_{i-l, j} b_{0}^{-l} \\
& =b_{0}\left(b_{j} b_{0}^{-1-i}-\sum_{m=1}^{i+1} a_{i+1-m, j} b_{0}^{-m}\right) \\
& =b_{0} \theta\left(s_{i+1, j}\right) .
\end{aligned}
$$

It follows that we have a ring homomorphism as described. It induces a map $R[1 / e] \rightarrow R^{\prime}$, which we again call $\theta$. On the other hand, it is clear that there is a unique map $\sigma: R^{\prime} \rightarrow R[1 / e]$ sending $b_{i}$ to $b_{i}$, and that $\theta \sigma=1$. As $s_{j+1, k}=\left(s_{j k}-a_{j k}\right) / e$ in $R[1 / e]$, we see inductively that $s_{j k}$ lies in the image of $\sigma$ for all $j$ and $k$. A similar argument shows that $t_{k}$ lies in the image of $\sigma$, so $\sigma$ is surjective. As $\theta \sigma=1$ we deduce that $\theta$ and $\sigma$ give an isomorphism $R[1 / e] \simeq R^{\prime}$.

Lemma 8 The following diagram commutes:

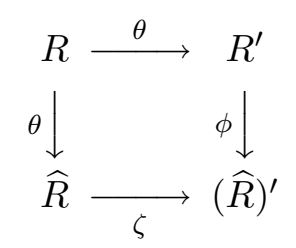

Proof First, recall that $\xi\left(b_{j}\right)$ is the coefficient of $x^{j}$ in the series $e+_{F} x=$ $\sum_{m, j} a_{m j} e^{m} x^{j}$, so $\xi\left(b_{j}\right)=\sum_{m=0}^{\infty} a_{m j} e^{m}$. Next, we have $\theta\left(s_{i j}\right)=b_{j} b_{0}^{-i}-$ $\sum_{l=1}^{i} a_{i-l, j} b_{0}^{-l}$. By putting $m=i-l$ we can rewrite this as

$$
b_{j} e^{-i}-\sum_{m=0}^{i-1} a_{m j} e^{m-i}
$$

It follows that $\xi \theta\left(s_{i j}\right)=\sum_{m=i}^{\infty} a_{m j} e^{m-i}=\sum_{k=0}^{\infty} a_{i+k, j} e^{k}$. By inspecting Proposition 6 , we see that this is the same as $\zeta \phi\left(s_{i j}\right)$.

Next, recall that $\theta\left(t_{k}\right)=-\sum_{l=1}^{k} b_{k-l} e^{-l}$, so

$$
\begin{aligned}
\xi \theta\left(t_{k}\right) & =-\sum_{l=1}^{k} \sum_{m=0}^{\infty} a_{m, k-l} e^{m-l} \\
& =-\sum_{r} e^{r-k} \sum\left\{a_{i j} \mid i+j=r, 0 \leq i<k, 0 \leq j\right\}
\end{aligned}
$$

Geometry \& Topology, Volume 5 (2001) 
(by putting $r=m-l-k$ and $j=m$ and $i=k-l$ and noting that $a_{i j}=a_{j i}$ ). Next observe

$$
\sum_{r} e^{r-k} \sum\left\{a_{i j} \mid i+j=r, 0 \leq i, j\right\}=e^{-k}[2](e)=0 \text { in }(\widehat{R})^{\prime} .
$$

It follows that

$$
\xi \theta\left(t_{k}\right)=\sum_{r} e^{r-k} \sum\left\{a_{i j} \mid i+j=r, i \geq k, j \geq 0\right\} .
$$

On the other hand, we have

$$
\zeta \phi\left(t_{k}\right)=\sum_{i, j \geq 0} a_{i+k, j} e^{i+j}
$$

This is the same up to reindexing, as required.

\section{$6 \quad e-$ power torsion}

Recall that $\left(1+t_{1}\right) e=0$ in $R$. We shall check that this gives all the $e$-power torsion, or equivalently that $e$ is a regular element in the ring $\bar{R}=R /\left(1+t_{1}\right)$. Our method is to exhibit $\bar{R}$ as a colimit of rings $\bar{R}_{k}$ in which the corresponding fact can be checked directly.

Proposition 9 The element $e$ is regular in $\bar{R}$.

Proof For $k>1$ we put

$$
\begin{aligned}
& A_{k}=L\left[t_{k}, s_{k j} \mid 0<j\right] \\
& B_{k}=L\left[t_{i}, s_{i j} \mid 0 \leq i \leq k, 0<j\right] .
\end{aligned}
$$

We define a polynomial $g_{k}(e) \in A_{k}[e]$ by

$$
g_{k}(e)=\sum_{l, m=0}^{k-1} a_{m l} e^{m+l-1}+\sum_{l=1}^{k-1} s_{k l} e^{k+l-1}+t_{k} e^{k-1} .
$$

We then put $\bar{R}_{k}=A_{k}[e] / g_{k}(e)$. One checks that $g_{k}(0)=2$, which is a regular element in $A_{k}$. It follows easily that $g_{k}(e)$ is regular in $A_{k}[e]$, and thus that $e$ is regular in $\bar{R}_{k}$.

Geometry 83 Topology, Volume 5 (2001) 
Next, we define a map $\pi: B_{k} \rightarrow A_{k}[e]$ by

$$
\begin{aligned}
\pi\left(t_{i}\right) & =\sum_{l=0}^{k-1-i} \sum_{m=0}^{k-1} a_{m, i+l} e^{m+l}+\sum_{l=0}^{k-1-i} s_{k, i+l} e^{k+l}+t_{k} e^{k-i} \\
\pi\left(s_{i j}\right) & =\sum_{l=0}^{k-1} a_{i+l, j} e^{l}+s_{k j} e^{k-i} .
\end{aligned}
$$

In the case $j=0$ of the second equation, the term $s_{k 0}$ is to be interpreted as 0 . It is easy to see that $\pi\left(t_{k}\right)=t_{k}$ and $\pi\left(s_{k j}\right)=s_{k j}$ and $\pi\left(s_{00}\right)=e$, so the notation is consistent. Moreover, we see that $\pi\left(1+t_{1}\right)=g_{k}(e)$. Using this, we see that $\pi$ induces an isomorphism $B_{k} / I_{k} \rightarrow \bar{R}_{k}$, where

$$
\begin{aligned}
I_{k}= & \left(t_{0}, t_{1}+1, s_{10}-1\right)+ \\
& \left(s_{i 0} \mid i>1\right)+ \\
& \left(t_{i}-s_{0 i}-s_{00} t_{i+1} \mid 0 \leq i<k\right)+ \\
& \left(s_{i j}-a_{i j}-s_{00} s_{i+1, j} \mid 0 \leq i<k, 0 \leq j\right) .
\end{aligned}
$$

It is clear from this that $\bar{R}=\lim _{\rightarrow} \bar{R}_{k}$. As $e$ is regular in $\bar{R}_{k}$ for all $k$, it must be regular in $\bar{R}$ as well.

Corollary 10 The e-power torsion in $R$ is generated by $t_{1}+1$, and thus is annihilated by $e$.

Proof Suppose that $u \in R$ and $e^{k} u=0$ for some $k>0$. It is clear from the proposition that the image of $u$ in $\bar{R}$ must be zero, so $u \in\left(t_{1}+1\right)$, so $e u=0$.

Corollary 11 There is a pullback square of rings as follows:

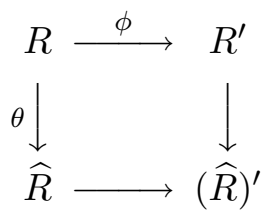

Thus, $R=M U_{A}^{*}$.

Proof Combine Corollary 10, Theorem 4, Proposition 6, Proposition 7, Lemma 8 , and Theorem 3.

Geometry \& Topology, Volume 5 (2001) 


\section{References}

[1] J P C Greenlees, J P May, Generalized Tate Cohomology, volume 113 of Memoirs of the American Mathematical Society, Amer. Math. Soc. (1995)

[2] I Kriz The $\mathbf{Z} / p$-equivariant complex cobordism ring, from: "Homotopy invariant algebraic structures (Baltimore, MD, 1998)", Amer. Math. Soc. Providence, RI (1999) 217-223

[3] L G Lewis, J P May, M Steinberger, (with contributions by Jim E McClure), Equivariant Stable Homotopy Theory, volume 1213 of Lecture Notes in Mathematics, Springer-Verlag, New York (1986)

[4] DP Sinha, Computations of complex equivariant bordism rings, e-print arxiv:math.AT/9910024 